\title{
Influence of age on postural control during dual task: a centre of pressure motion and electromyographic analysis
}

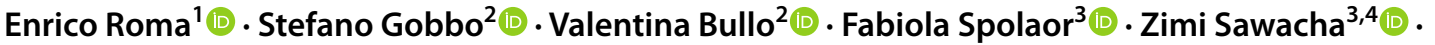

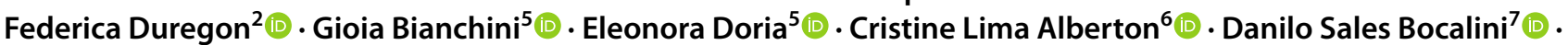

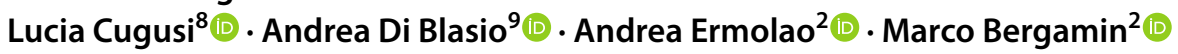

Received: 8 November 2020 / Accepted: 13 May 2021 / Published online: 11 June 2021

(c) The Author(s) 2021

\begin{abstract}
Background Dual task influences postural control. A cognitive task seems to reduce muscle excitation during a postural balance, especially in older adults (OA).

Aim The aim of this study is to evaluate the effect of three cognitive tasks on muscle excitation and static postural control in OA and young adults (YA) in an upright posture maintenance task.

Methods 31 YA and 30 OA were evaluated while performing a modified Romberg Test in five different conditions over a force plate: open eyes, closed eyes, spatial-memory brooks' test, counting backwards aloud test and mental arithmetic task. The surface electromyographic signals of Tibialis anterior (TA), Lateral Gastrocnemius (GL), Peroneus Longus (PL), and Erector Spinae (ES) was acquired with an 8-channel surface electromyographic system. The following variables were computed for both the electromyographic analysis and the posturographic assessment: Root mean square (RMS), centre of pressure (CoP) excursion (Path) and velocity, sway area, RMS of the CoP Path and 50\%, 95\% of the power frequency. Mixed ANOVA was used to detect differences with group membership as factor between and type of task as within. The analysis was performed on the differences between each condition from OE.

Results An interaction effect was found for Log (logarithmic) Sway Area. A main effect for task emerged on all posturographic variables except Log 95\% frequencies and for Log PL and ES RMS. A main effect for group was never detected.

Discussion and conclusion This study indicates a facilitating effect of mental secondary task on posturographic variables. Non-silent secondary task causes increase in ES and TA muscle activation and a worsening in static postural control performance.
\end{abstract}

Keywords Balance $\cdot$ Cognition $\cdot$ Fall $\cdot$ Elderly $\cdot$ Stabilometry $\cdot$ Emg

Stefano Gobbo

stefano.gobbo@unipd.it

1 Laboratoire Interuniversitaire de Biologie de la Motricité (LIBM), EA 7424, Univ Lyon, UJM Saint-Etienne, 42023 Saint-Etienne, France

2 Sport and Exercise Medicine Division, Department of Medicine, University of Padova, Via Giustiniani, 2, 35128 Padova, Italy

3 Department of Information Engineering, University of Padova, Via Gradenigo, 6, 35121 Padua, Italy

4 Department of Medicine, University of Padova, Via Giustiniani, 2, 35128 Padova, Italy

5 GymHub S.R.L., Spin-off of the University of Padova, Via O. Galante 67/a, 35129 Padova, Italy
6 Physical Education School, Federal University of Pelotas, Rua Luís de Camões, 625, Pelotas, RS 96055630, Brazil

7 Laboratorio de Fisiologia e Bioquimica Experimental, Centro de Educacao Fisica E Deportos, Universidade Federal do Espirito Santo (UFES), Av. Fernando Ferrari, 514, Goiabeiras, Vitoria, ES, Brazil

8 Department of Biomedical Sciences, University of Sassari, Viale San Pietro 43/B, 07100 Sassari, Italy

9 Department of Medicine and Sciences of Aging, G. D’Annunzio University of Chieti-Pescara, Via dei Vestini, 31, 66013 Chieti, Italy 


\section{Introduction}

Postural control is a complex motor skill that results from the interaction between biomechanical constraints, movement and sensory strategies, orientation in space and cognitive processing [1]. Even if static postural control is an automatic process [2], it can require a certain level of attention in dual task condition [3-5]. If a secondary task is carried out concurrently with the postural one, attentional resources will be divided [6-9].

Performing two tasks at the same time is defined as dual task (DT) [10]. Dual-task cost (DT cost) occurs when the DT paradigm includes two tasks (e.g., a motor task and a cognitive one) [11] and it is measured as a deviation from a single task (ST) performance. Task prioritization depends on type of tasks, goal and instruction given to participants, defining concept of postural reserve and hazard estimation [12]. Older adults (OA) tend to perform worse than young adults (YA) under DT condition [13, 14], especially in the postural task [10]. However, part of the explication is that postural sway has been shown to increase with ageing [13], showing a worsening in static postural control. If the postural task is not threating, prioritization will favour the concurrent one [15]. Increasing difficulty of postural task enhances its DT cost but does not cause a reduction in performance of the cognitive concurrent task [16]. The present phenomena can be partially explained by bottleneck theory [17]. Moreover, degenerative processes that culminate in cognitive impairment could explicate reduction of attentional resources in older adults [10]. It has been demonstrated that OA are unable to select relevant information present in the environment, using all of them [18]. However, there is no consensus on how this could affect postural control $[13,14,19]$. Older Adults attempt to decrease postural sway by increasing ankle stiffness to counteract the inability to quickly respond to perturbations [5, 16, 19-21], by increasing co-contraction of agonist-antagonist muscles of the lower leg [5] and by a tighter neuromuscular control [16, 22]. The first mechanism could be considered as a strategy to make postural control more automatic [20,21], even if they are energetically inefficient [23]; the second one requires attentional resources $[16,22]$.

Previous research used surface electromyography (sEMG) to evaluate postural control under DT condition [24-27]. A cognitive task seems to reduce muscle activity quantified as normalized signal amplitude during an ankle movement task [26] and a balance recovery one [25]. However, cocontraction index seems not to vary between conditions in adult healthy participants [27]. Fatigue can reverse previous relationship, in fact after a workload, muscle activity under
DT increases [24]. On the contrary, difficult of the cognitive task did not influence sEMG parameters [26]. In OA reduction in sEMG magnitude is more pronounced than in their younger counterpart [25] in both agonist and antagonist muscles [25], suggesting that less attentional resources for balance are available.

The effect of different types of cognitive tasks on muscle excitation and DT cost remains understudied. Moreover, heterogeneity between postural balance tasks and their difficulties can hide the true effect. Thus, the aim of this study is to evaluate the effect of different types of cognitive tasks on muscle excitation and static postural control in young and older adults in an upright posture maintenance task. Our hypothesis is that an interaction effect between age and type of task would be present for stabilometric and muscle excitation variables.

\section{Material and methods}

\section{Participants}

The Sport and Exercise Medicine Division, University of Padova provided two databases for sample recruitment of $\mathrm{OA}$ and YA. Records were selected according to a database specific computer-generated list of random numbers and then potential participants were contacted by phone and they were asked to participate in an anamnesis session. Exclusion criteria were: (i) uncorrected visual impairments, (ii) lower limbs orthopaedic surgery in the 6 months prior to the study, (iii) use of medication that could influence postural control, (iv) neurological disorders, (v) cognitive impairments, vi) history of falls in the previous 6 months. Moreover, participants were eligible if they were able to stand upright for 10 minutes without supports (self-reported). At the end of this process, $31 \mathrm{YA}$ (17 women, age $22 \pm 4 \mathrm{yr}$, range 18-26) and $30 \mathrm{OA}$ (19 women, age $72 \pm 4$, range $65-84$ ) were enrolled to take part to the study. All participants completed an informed consent process before starting experimentation. Sample size was selected according with Bergamin et al. [13]. In the first session, one researcher administered a medical history questionnaire and the Mini-Mental State Examination (MMSE) [28]. Cut off value for MMSE was 27. In eligible participants, height and body mass were measured.

\section{Procedure}

In the second session participants carried out the following tasks, for $30 \mathrm{~s}$ each: open (OE) and closed (CE) eyes, 
spatial-memory brooks' test (SMBT) [29], counting backwards aloud test (CBAT) and mental arithmetic task (MAT) [21]. Participants were asked to stay as still as possible [30] while performing a modified Romberg Test [31]: upright stance, gaze at the target on the wall, arms along the body, joined heels and tips of the feet spread apart of thirty degrees. Before starting, the target was set at eye level. The order of experimental conditions was randomized to prevent learning effect. Five minutes rest between conditions was planned to reduce potential fatigue effect. Three trials for each condition were performed and the mean value for each variable was considered.

\section{Experimental dual tasks}

\section{Spatial-memory Brooks' test}

Before starting, an empty 4 by 4 matrix with only the number one in position 2,2 was shown. A computerized audio track, randomly selected from 12 available, indicated the spatial placing for number from 1 to 8 . Numbers can be placed to the right, left, up or down respect to the previously filled square, but they cannot overlap themselves or be place outside the matrix. At the end of the trial participants had to fill the matrix with the numerical sequence. Two familiarization attempts were planned before procedure began. Individual score was determined counting correct answers, maximal score was 7 .

\section{Counting backwards aloud test}

Participant was asked to count backward aloud by one, as fast and as accurately as possible, with a clear pronunciation from a randomly selected number from 90 to 100 . Individual score was determined by counting mistakes [32].

\section{Mental arithmetic task}

A computerized audio-track enunciated nine arithmetic operations mixed between additions and subtractions with a pause of $2 \mathrm{~s}$ between them. A software randomly selected the digit sequence so that partial and final algebraic sums were always positive natural numbers. Participants were asked to mentally and wordlessly solve numerical series and to return the result of the trial. Score was a binary variable, "correct" or "wrong".

\section{Outcomes assessments}

\section{Electromyography recording and processing}

After appropriate cleaning and preparation of the skin sEMG probes (FREEEMG, BTS Bioengineering, Padova) were applied bilaterally, according with Blanc's guidelines [33], over Tibialis anterior (TA), Lateral Gastrocnemius (GL), Peroneus Longus (PL), Erector Spinae Longissimus (ES). The 8-channel surface electromyographic system was used with a frequency of acquisition of $1000 \mathrm{~Hz}$ for each channel. Signals were band pass filtered between 10 and $450 \mathrm{~Hz}$ with a 5th order Butterworth filter and full wave rectified. The root mean square (RMS) of the signal over the entire trial (on a $50 \mathrm{~ms}$ window) was computed by low-pass filtering the signals with a 4th order Butterworth filter and a cut off frequency of $5 \mathrm{~Hz}$ [34]. The mean RMS between left and right leg muscles and left and right ES muscles were used. To allow comparisons results are expressed as percentage of the muscle activity during OE condition [35]. Data were processed using Matlab (MATLAB and Statistics Toolbox Release 2012b, The MathWorks, Inc., Natick, Massachusetts, United States).

\section{Stabilometric variables recording and processing}

Ground reaction forces were recorded by a force platform (FP4060-10, Bertec Corporation, Columbus, OH) with a sampling frequency of $960 \mathrm{~Hz}$, fixed to the floor $3 \mathrm{~m}$ away from walls and $5 \mathrm{~m}$ from the target. Foot placement was standardized as recommended by previous findings $[31,36]$. A set of parameters was chosen according with the literature [36-38]. The following variables were taken for the analysis: center of pressure (CoP) excursion (path), CoP velocity (CoPv), sway area (SA)[31], root mean square of CoP displacement (RMS) and 50\% and $95 \%$ of power frequency [31]. $\mathrm{CoPv}$ was defined as the mean velocity of $\mathrm{CoP}$ during the entire trial. The SA estimate the area enclosed by the CoP path per unit of time, calculated summing up the area of the triangles formed by two consecutive points on the CoP path and the mean CoP [31]. The frequency, below which $50 \%$ and $95 \%$ of the total power frequency was found, are indicated as 50\% and 95\% frequency. The RMS represent the standard deviation respect to the mean displacement of the CoP [31]. Velocity, displacement and frequencies measures components along anterior-posterior (z) and medio-lateral (x) axis are reported. Data were processed using Matlab 
Log Path

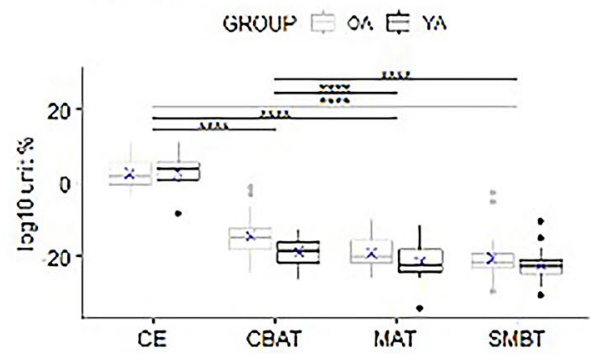

Log Path z

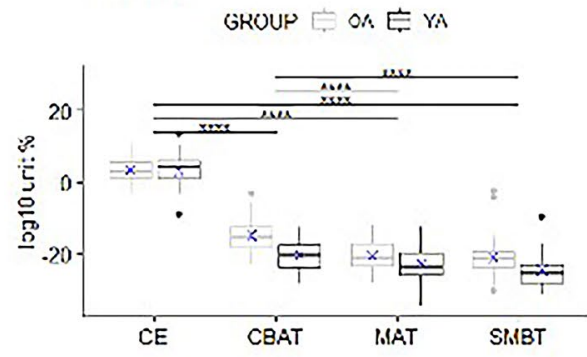

Log Mean Velocity

GROUP EOA

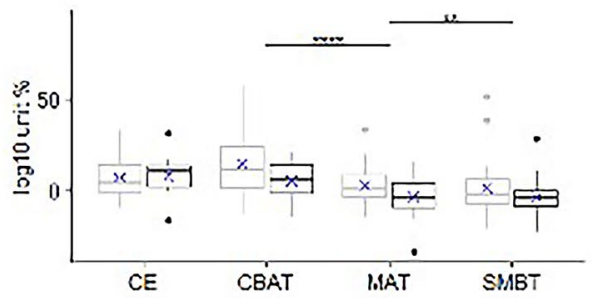

Log Mean Velocity z

GROUP On E $\mathrm{YA}$

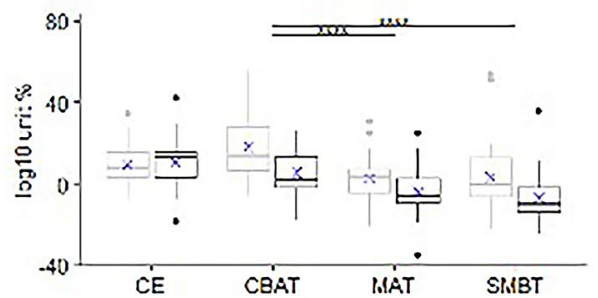

Log Median Frequencies z

GROUP $\dot{H}$ OA

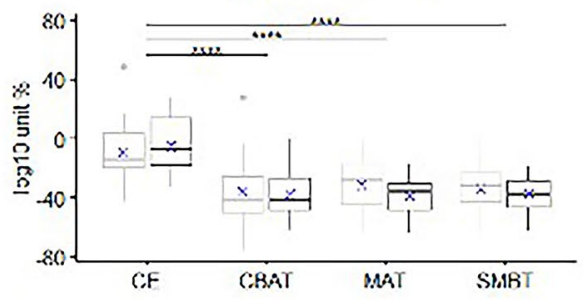

$\log P a t h x$

GROUN $\exists$ O. 白 $\mathrm{m}$

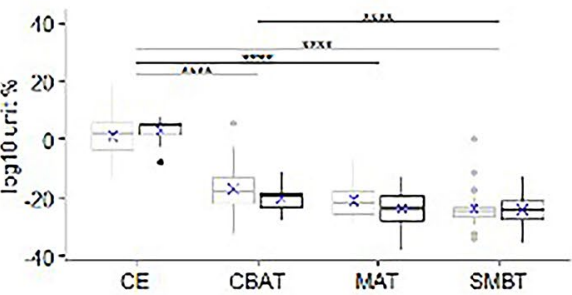

Log RMS

GROUH $\exists$ an $\boxminus Y_{\text {M }}$

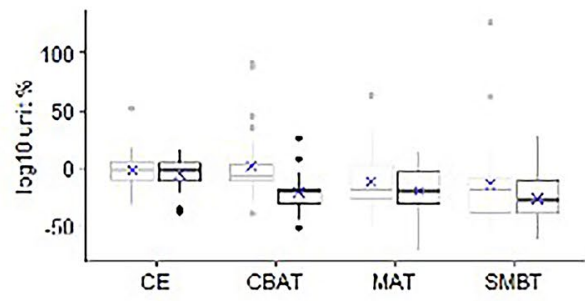

Log Mean Velocity $x$

GROUN $\exists$ on $\boxminus Y_{N}$

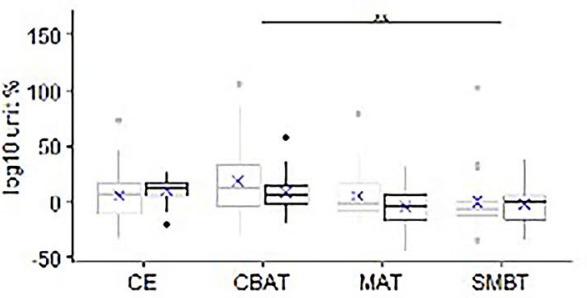

Log Median Frequencies $x$

GROUN $\exists$ an $\Leftrightarrow \mathrm{G}$

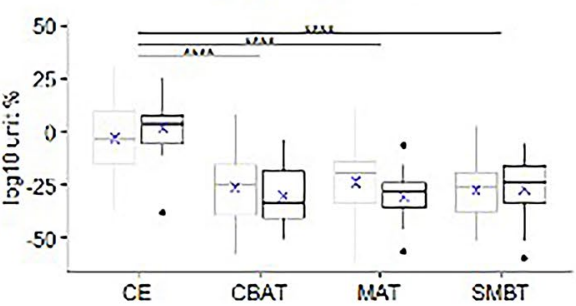

Log Sway Area

GROUP C: G

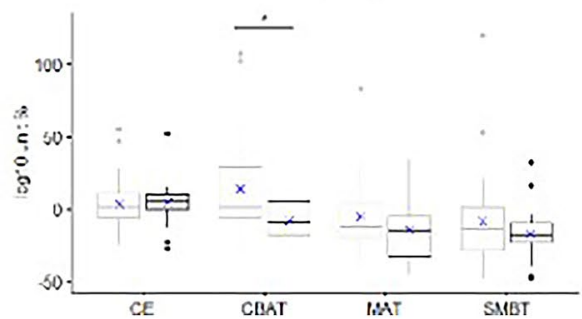


\Fig. 1 Boxplots with superimposed the mean $(X)$ for stabilometric variables. In the sway area graph, only the significance within CBAT condition is reported to not add confusion to the figure. For the complete results of this variable, please refer to the homonymous section. $*, p<0.05, * *, p<0.01$, ***, $p<0.001$, ****, $p<0.0001$. OA Older Adults, $Y A$ : Young Adults, $C E$ closed eyes, $C B A T$ counting backward aloud test, MAT mental arithmetic task, $S M B T$ spatial memory Brooks test

(MATLAB and Statistics Toolbox Release 2012b, The MathWorks, Inc., Natick, Massachusetts, United States). The force plate was synchronized with the dual task audio track to allow for an exact $30 \mathrm{~s}$ duration of the task and recording.

\section{Statistical analysis}

Sociodemographic characteristics were compared through T-test. Chi-square was used to compare results of the cognitive task (treated as dichotomous) and group composition.

Due to highly skewed distributions, the based-10 logarithm $(\log )$ was calculated for each variable. To assess the need for correction for anthropometric characteristics, stabilometric parameters were plotted against height and body mass. Visual analysis and a significant Pearson product moment correlation with $r>0.7$ were used as criteria for normalization. No one of the parameters met the requirement for correction.

The percentage differences between the Log values of each condition (CE, CBAT, MAT, SMBT) and control one $(\mathrm{OE})$ were computed and used for the statistical analysis. Normality of the data was assessed via Q-Q plot and Shapiro-Wilk. Mild violations of this assumption were allowed due to the robustness of the model. Two-way split-plot ANOVA was used with group membership as between subject factor (2 levels) and type of task as within factor (5 levels). Box test was used to check equality of co-variance matrix with an $\alpha=0.01$. For all ANOVAs, sphericity was tested with the Mauchly test for the dependent variables within group analysis. If the assumption of sphericity was violated, the correction of Greenhouse-Geisser was applied. Levene's test was used to check the assumption of equality of variances for between group analyses. The presence of outliers was evaluated via residuals. To follow-up significant interaction effect, repeated measure ANOVA was used to test for main effect for task within each group and independent $T$ test was used to test main effect for group. Effect size for any main factor and interaction was calculated and presented by partial $\eta^{2}\left(\eta^{2} p\right)$.

We decided to apply Bonferroni's correction only to pairwise comparisons to follow up a significant main effect for condition. Hence, we reported the corrected values. However, as suggested by one of the reviewers, we calculated the Bonferroni's level of significance accounting for 139 tests (45 Fisher tests and 94 pairwise comparisons), that is 0.00037 . We will discuss the finding accounting this value for the calculation, though we are not using it as a binary criterion to determine significance. For this reasons, exact $p$ value are reported.

Statistical analysis was performed with R [R Core Team (2018)]. Results are expressed as mean (SD).

\section{Results}

In the present section, we are using the abbreviation of the experimental condition (CE, CBAT, MAT, SMBT) to indicate the percentage differences of the Log raw values respect to $\mathrm{OE}$ condition.

\section{Sociodemographic characteristics and cognitive performance}

The OA group was composed by 30 participants (19 women, $63 \%$ ) and the YA group by 31 (17 women, 55\%). The height was significantly higher in YA than OA. Moreover, OA has a significantly higher BMI than YA. Groups did not differ on cognitive performance. Sociodemographic characteristics and cognitive performance error counts are exposed in Table 1.

\section{Stabilometric analysis}

The results of split-plot ANOVA on posturographic variables are extensively reported in Table 2 . There was a statistically significant interaction between tasks and groups only for Log Sway Area $\left(F_{(2.55,106.92)}=4.03, p=0.01\right.$ $\left.\eta^{2} p=0.09\right)$. Following up main effects, it emerged that OA (mean $=-8.79, \mathrm{sd}=16.69)$ differed significantly from YA (mean $=13.86, \mathrm{sd}=33.66)$ only in the CBAT condition $\left(t_{(46)}=3.491, p=0.01, d=0.209\right)$. Moreover, a significant effect for task was present for both, the OA $\left(F_{(2.33,55.85)}=10.71, p<0.0001, \eta^{2} p=0.31\right)$ and the YA 


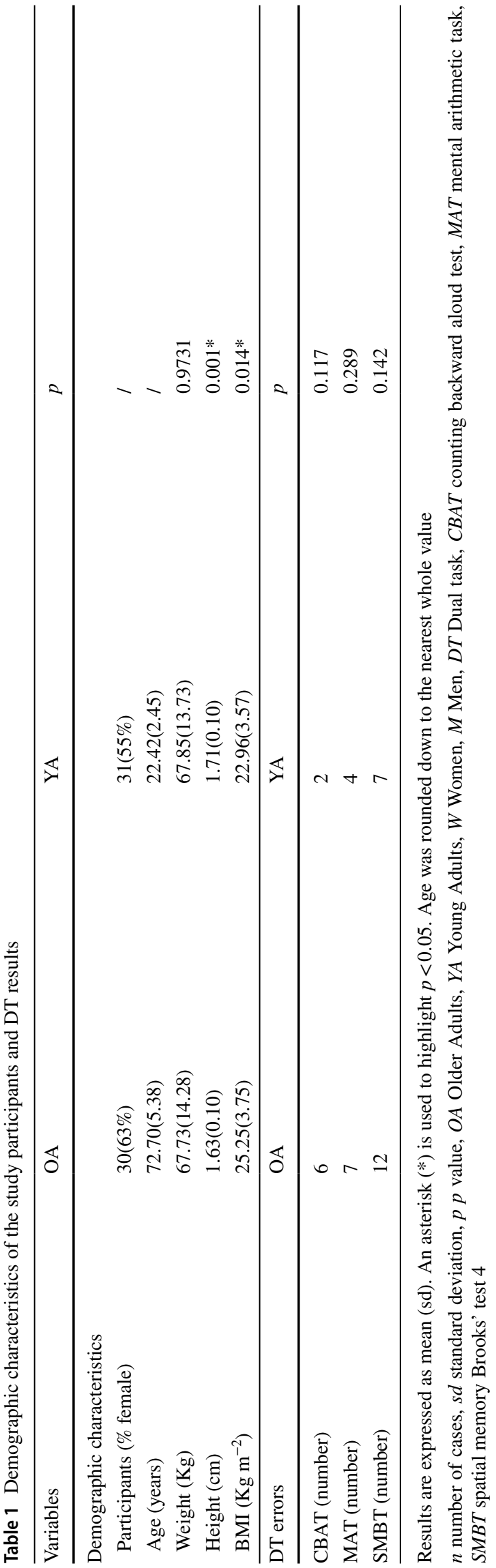

$\left(F_{(3,54)}=7.03, p=0.0005, \eta^{2} p=0.28\right)$. For the OA, there was statistically significant differences between: CBAT vs MAT $(p=0.011)$, CBAT vs SMBT $(p=0.0002)$. For the YA, there was statistically significant differences between: CE vs MAT $(p=0.003)$, CE vs SMBT $(p=0.002)$. A main effect for task emerged for all variables; Table 3 reports pairwise comparisons (see Fig. 1). Only Log $95 \%$ frequencies z showed a main effect for group $\left(F_{(1,53)}=4.47, p=0.04 \eta^{2} p=0.08\right)$. In all the DT condition, OA had a greater negative difference (respectively, $p=0.002, p=0.04, p=0.02$ ) from control condition if compared to YA.

\section{EMG analysis}

Statistical interaction between age and condition was not found in the considered muscle, as main effect for age group. However, TA and ES showed a main effect for condition, respectively $F_{(2.04,116.45)}=8.26, p=0.0004 \eta^{2} p<0.01$, and $F_{(3,168)}=6.29, p=0.0005, \eta^{2} p=0.01$. Table 3 reports pairwise comparisons (see Fig. 2).

\section{Discussion}

\section{Main findings}

This study aimed to evaluate the effect of different types of cognitive tasks on postural control and muscle excitation of the lower limb and the trunk, between OA and YA. It was hypothesized that interaction effect should be present between age and type of secondary task in all the variables, however the main finding is that interaction occurred only for $\log$ SA. The portion of variance explained by interaction, excluding the main effect, is $9 \%\left(\eta^{2} p=0.09\right)$. Moreover, the $p$ value for interaction does not exceed the Bonferroni's level of significance ( $p=0.01$ vs $p=0.00037$ ). Type of task seems to act in an independent manner, disregarding the age of participants. These results were in agreement with previous findings: OA were able to manage the complexity of DT as the younger people in a stationary condition [13, 14], despite a dynamic secondary task would have shown a better discriminant capacity, being more challenging for the postural control system [39].

However, considering that, Bonferroni adjustment tends to overcorrect the level of significance and that multiple authors are against its use [40], our intent is to explain why the interaction was occurring. The presence of this effect was justified by the fact that OA increased their SA respect to control condition during CBAT and decreased it during MAT and SMBT. On the contrary, SA in young adults increased during CE, and differed from MAT and SMBT. One possible reason could be that verbalization increased respiratory acts, influencing $\mathrm{CoP}$ displacement [41]. In 


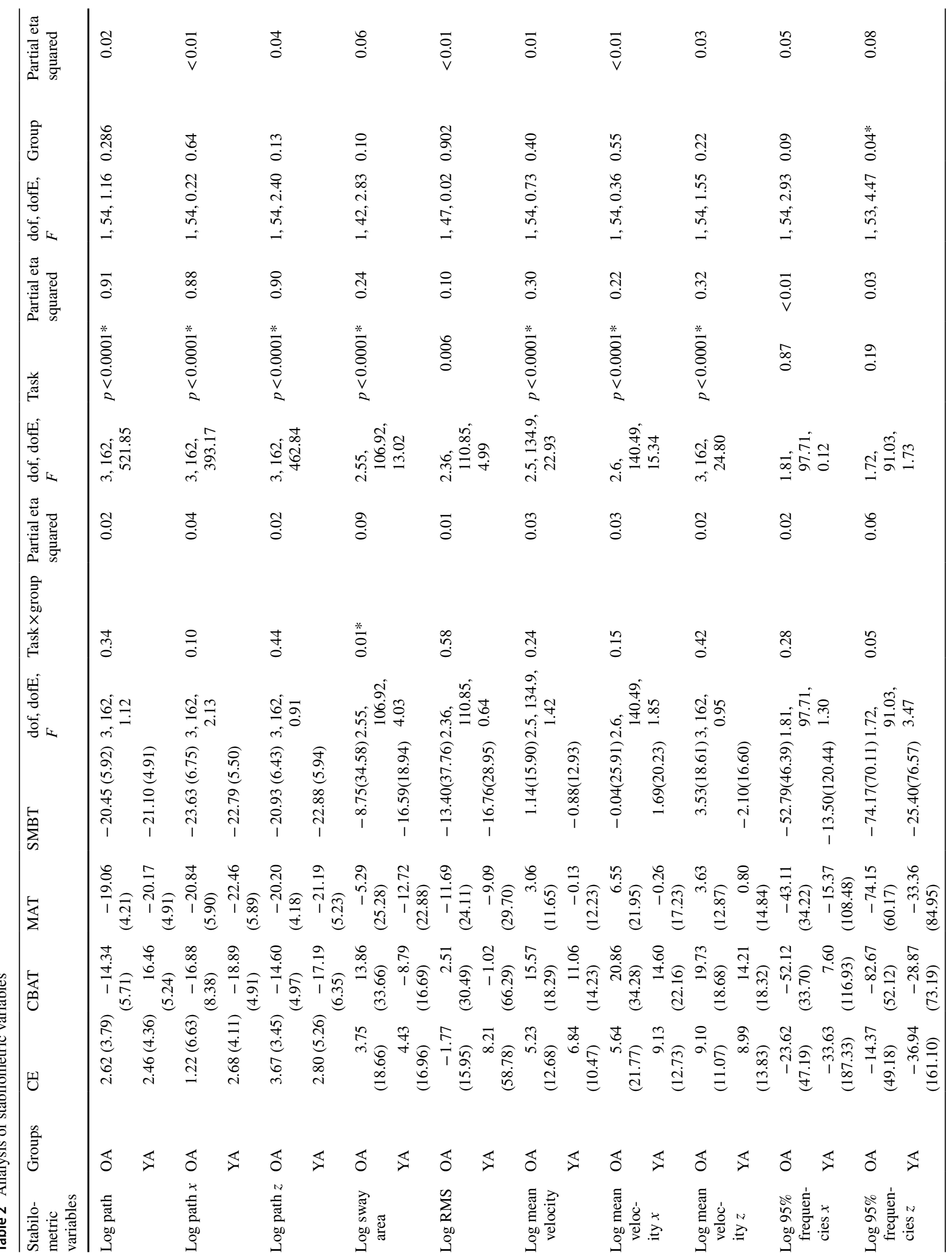




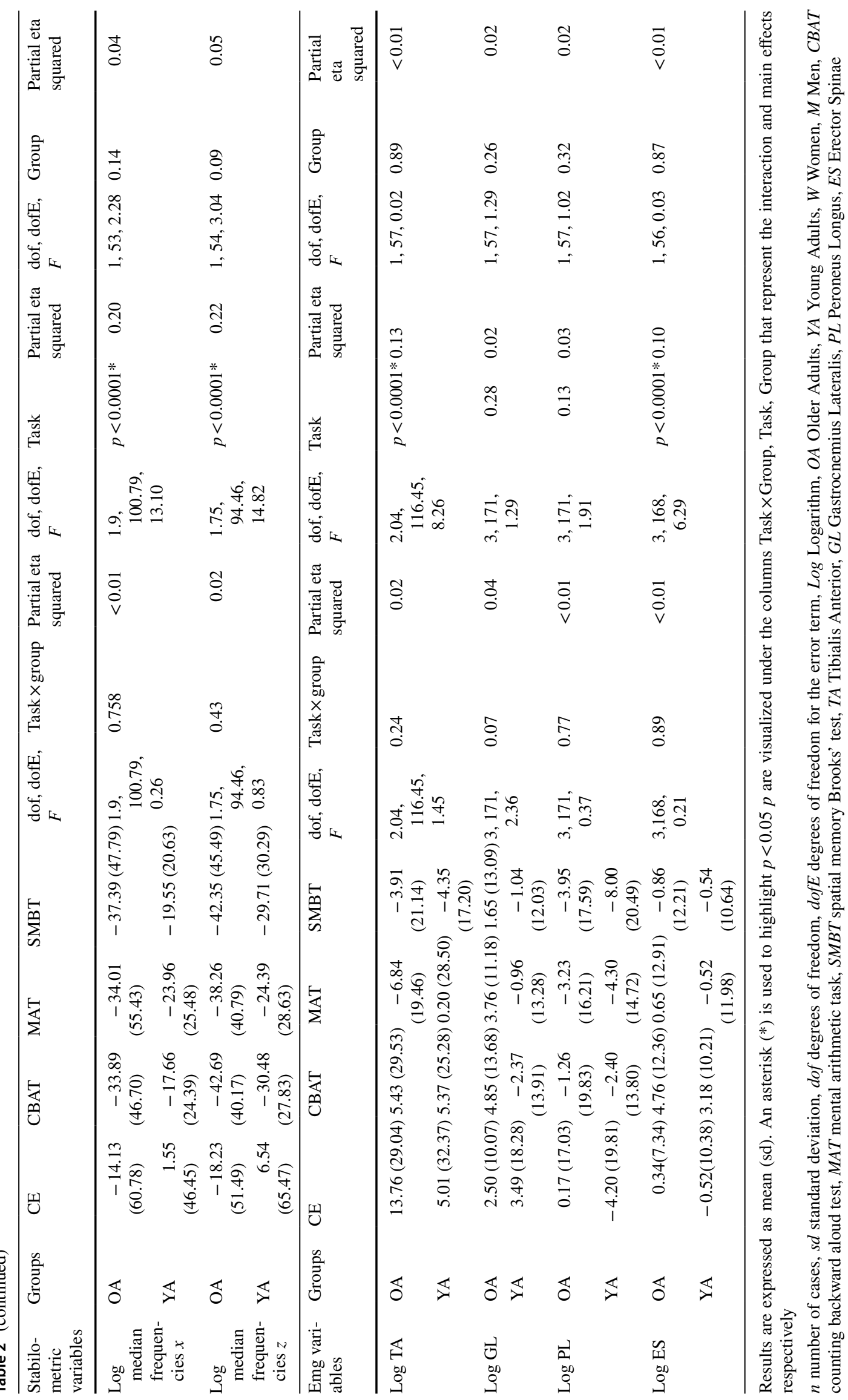




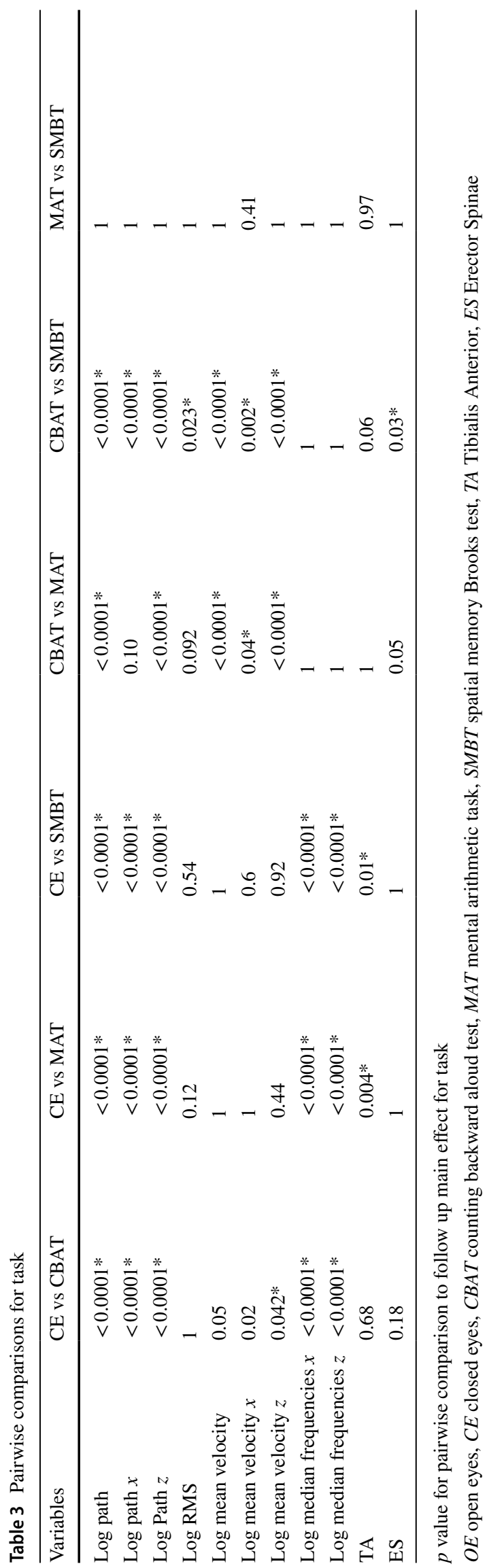

support to this hypothesis, we reported significantly higher ES muscle excitation in CBAT respect SMBT (the difference between CBAT and MAT approximate significance), similarly to the augmented ES muscle excitation found by Hodges et al. [41] during increased tidal volume condition. Generally, OA tends to respond to perturbation increasing co-contraction [5]. Whereas DT interferes with this mechanism, postural sway will be over-increased and interaction effect detected in SA could explain this response. Co-contraction is a function of agonist-antagonist muscle excitation [42]. As GL activity did not vary according to the task ( $p=0.28)$ while TA did $(p=<0.0001)$, it could be speculated that co-contraction in the lower leg was reduced. In considering findings of muscle activity, it is necessary to consider that even if the part of variance explained is low, they are quite consistent as they reach significance even after the correction for family-wise error rate. In agreement with our results, others reported a reduction of TA excitation during DT [26], but without difference when increasing the task complexity. However, it has to be considered that types of tasks were extremely different, as participants in the reference study performed an ankle movement while sitting.

The authors of this paper were not able to explain why the interaction was occurring only for SA. Normally postural sway parameter accounts for a certain degree of redundancy $[43,44]$. Evaluating the mean of the parameter with a dimensional equation similar to SA, as mean velocity (and its components), you will see a similar behaviour. Given that, squaring the length component "increased" his importance in the computation. A second interpretation could be that that interaction is due to chance and parameter only present a main effect for task (as stated above in the first paragraph).

A significant main effect for task was always present in the stabilometric parameters except for Log $95 \%$ frequencies and Log RMS, with $\eta^{2} p$ ranging from 0.20 to 0.91. Considering that RMS is linked to total power of the spectrum, [43] it can explain the similar trend as $95 \%$ frequency. Time-domain parameters reflected partially the previous results of Bergamin et al. [13]. All parameters related to CoP path and RMS were significantly higher in ST than DT, even if the effect of task was more accentuated on path length and its components. A possible explication is that performing an external task forced it to be automatic (participants are no more concentrated to stand "as still as possible" trying to improve the static postural control performance [45]). According to previous literature, the instruction "stand as still as possible" improve reliability of the measure and the performance during the single task of postural control [30]. This phenomenon probably occurs because it reduces the degree of accommodation during "stand quietly" or no instruction trials. The DT conditions permits to obtain automaticity and no accommodation at the same time, improving 
$\log P L$

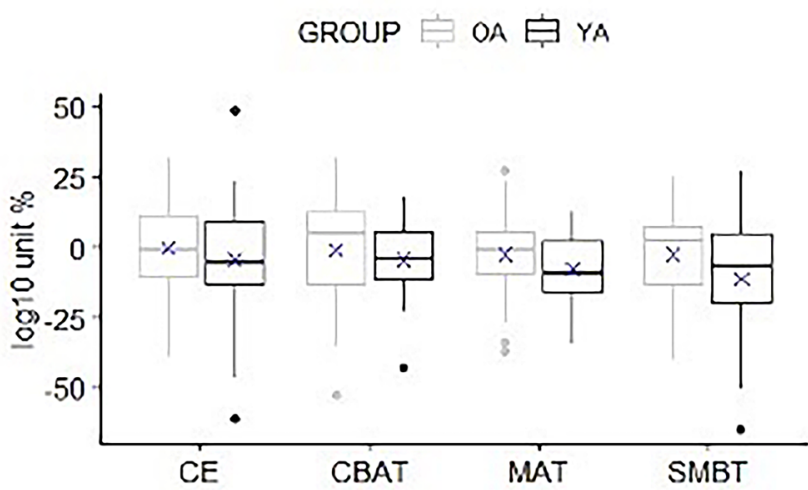

$\log T A$

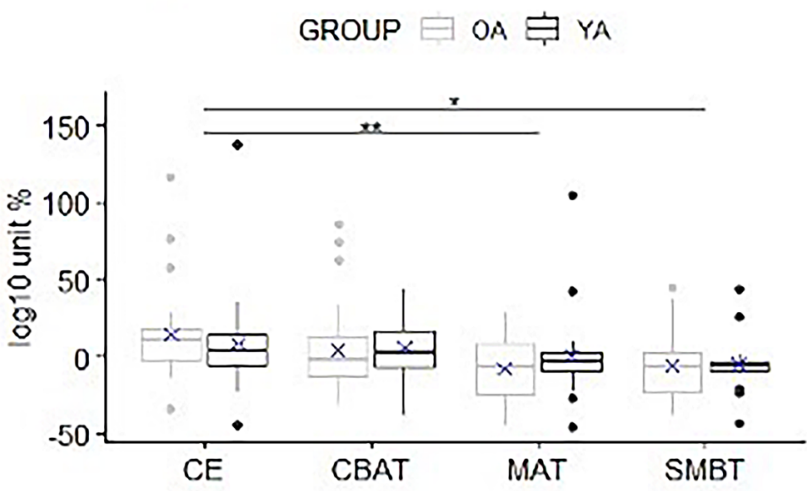

Fig. 2 Boxplots with superimposed the mean $(X)$ for electromyographical variables. *, $p<0.05, * *, p<0.01, * * *, p<0.001$, ****, $p<0.0001$. OA Older Adults, YA Young Adults, CE closed eyes,

the performance. From another perspective, performing a secondary task can produce undesired movements due to cognitive load, e.g., oculomotor activity [46], and the postural control system can organize itself to prevent these type of movements increasing co-contraction or acting to increase stability. Moreover, performing a secondary task has been demonstrated in reducing the exploratory movement of the feet [47].

Literature about frequencies showed that they could be useful to discern between visual, vestibular and somatosensory system actions [48]. In the present experiment, 95\% percent frequencies did not present a main effect for task or condition. Considering a potential role in discerning between postural control systems, the motivation can lie in the facilitating effect of DT. If participant balance condition were not threatened and if one system did not overcome the others, the parameter should not change. Moreover, considering this interpretation, our results showed agreement with those of Prieto et al. [49], meaning that at least the same set

\section{$\log G A L$}

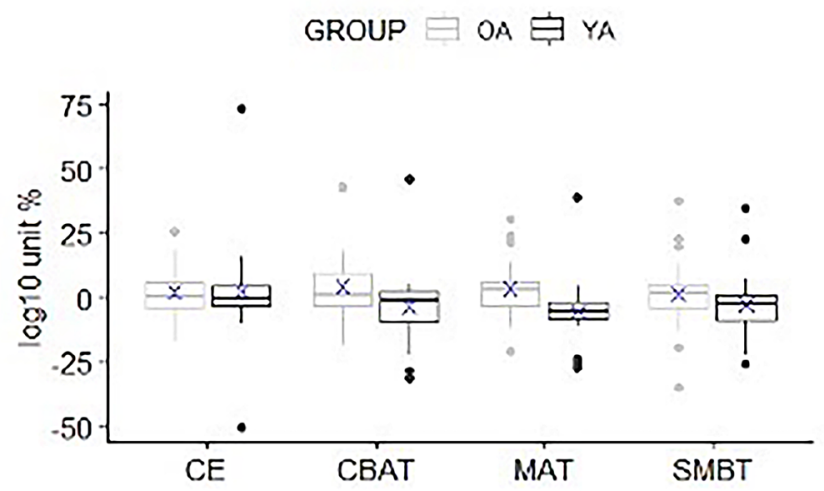

\section{Log ES}

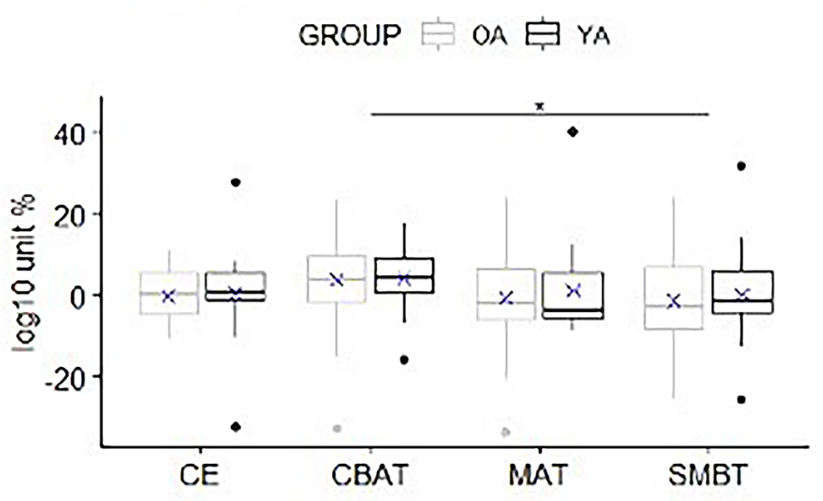

CBAT counting backward aloud test, MAT mental arithmetic task, $S M B T$ spatial memory Brooks test. TA Tibialis Anterior, GL Gastrocnemius Lateralis, $P L$ Peroneus Longus, ES Erector Spinae

of parameters should be used under DT conditions to better capture CoP characteristics.

In contrast with previous findings [49, 50], a main effect for group was found only for $\log 95 \%$. However, considering the $\mathrm{p}$ value and small effect size $\left(\eta^{2} p=0.08\right)$ we can assume that is negligible. This finding is not surprising as we computed the differences from the control condition, while the reported studies analyse the raw value. It is well documented in the literature that static postural control worsens with age, however OA and YA respond in a similar manner to the different task, if we account to the baseline.

About muscle activation, our results seemed to be in contrast with current literature which reported an increased activation in OA than YA [25]. However, that investigation analysed a balance recovery task while we analysed a balance maintenance task, and a direct comparison could generate misleading interpretations. Additionally, GL did not show any effect. This muscle is the main responsible for anterior-posterior oscillations [51] and according to 
prioritization and hazard estimation theory [12], postural control system could have maintained constant the muscle activity preventing the risk of fall.

In summary, DT affected participant's postural control and TA and ES muscle excitation, inducing a facilitating effect. An interaction effect was founded on SA in OA, tending to increase for CBAT condition but lowering during other DT respect to ST. Conversely, in YA, SA decreased in all DT conditions. Normalization to control condition eliminates all the other differences due to group membership. Muscle excitation did not show a homogeneous behaviour; in fact, GL and PL remained unaltered, while muscle activity changed for TA and ES.

\section{Limitations}

Stochastic parameters should be included to describe CoP displacement [52], but the longer testing time needed [38] could prevent the administration of five experimental conditions, potentially generating other bias (e.g., muscle fatigue in the elderly). Another potential criticism was that EMG normalization on OE condition was not equivalent to normalizing on the reference task for each muscle; however, during $\mathrm{OE}$ condition muscles work in near isometric manner and, in this way, results could be expressed as fraction of OE. Moreover, the normalization for OE condition was specific for the postural task performed during DT condition. With respect to EMG analysis, it should be mentioned that the detection of the timing of skeletal muscles activation prior to the extraction of any parameters from the EMG signal plays a significant role. Due to high signal-to-noise ratio required for accuracy in estimation, the detection of muscle activity becomes even more difficult [53] for myoelectric weak and noisy signals, such as those recorded during prolonged low-level sustained contractions (i.e., postural activity). In the present contribution a single threshold method was applied, which is more susceptible to the background noise and the environmental interferences. Asking to participant to stand as still as possible could be a limitation itself, creating a dual task condition in the reference task. However, using the "stand quietly" instruction may result in an accommodating posture due to lack of instructions.

\section{Conclusion}

The present study indicated that DT improves static postural control, probably increasing automaticity of the motor task. The most challenging experimental condition is CBAT, due to verbalization. Accounting for the baseline level, the performance of YA and OA varies in the same manner within the different condition for all the variables except sway area. Moreover, during a postural maintenance task muscle excitation is not different between OA and YA. Shank muscles showed a different response to experimental condition with only TA influenced by the DT, but not by task complexity. We can conclude that YA and OA tend to respond in the same manner to DT, exhibiting a facilitating effect in respect to single task condition.

Funding Open access funding provided by Università degli Studi di Padova within the CRUI-CARE Agreement. No funding was received to assist with the preparation of this manuscript.

\section{Declarations}

Conflict of interest The authors have no conflicts of interest to declare that are relevant to the content of this article.

Ethics approval The study complied with the current laws of Italy for research on human participants and it was approved by the local review board. This study was performed in line with the principles of the Declaration of Helsinki.

Informed consent All participants completed an informed consent process before starting experimentation.

Open Access This article is licensed under a Creative Commons Attribution 4.0 International License, which permits use, sharing, adaptation, distribution and reproduction in any medium or format, as long as you give appropriate credit to the original author(s) and the source, provide a link to the Creative Commons licence, and indicate if changes were made. The images or other third party material in this article are included in the article's Creative Commons licence, unless indicated otherwise in a credit line to the material. If material is not included in the article's Creative Commons licence and your intended use is not permitted by statutory regulation or exceeds the permitted use, you will need to obtain permission directly from the copyright holder. To view a copy of this licence, visit http://creativecommons.org/licenses/by/4.0/.

\section{References}

1. Horak FB (2006) Postural orientation and equilibrium: what do we need to know about neural control of balance to prevent falls? Age Ageing 35:ii7-ii11

2. Takakusaki K, Takahashi M, Obara K et al (2017) Neural substrates involved in the control of posture. Adv Robot 31:2-23

3. Fraizer EV, Mitra S (2008) Methodological and interpretive issues in posture-cognition dual-tasking in upright stance. Gait Posture 27:271-279

4. Huxhold O, Li S-C, Schmiedek F et al (2006) Dual-tasking postural control: aging and the effects of cognitive demand in conjunction with focus of attention. Brain Res Bull 69:294-305

5. Melzer I, Benjuya N, Kaplanski J (2001) Age-related changes of postural control: effect of cognitive tasks. Gerontology 47:189-194 
6. Doumas M, Krampe RT (2015) Ecological relevance determines task priority in older adults' multitasking. J Gerontol B Psychol Sci Soc Sci 70:377-385

7. Swanenburg J, De Bruin ED, Uebelhart D et al (2009) Compromising postural balance in the elderly. Gerontology 55:353-360

8. Woollacott M, Shumway-Cook A (2002) Attention and the control of posture and gait: a review of an emerging area of research. Gait Posture 16:1-14

9. Zijlstra A, Ufkes T, Skelton D et al (2008) Do dual tasks have an added value over single tasks for balance assessment in fall prevention programs? A mini-review. Gerontology 54:40-49

10. Ruffieux J, Keller M, Lauber B et al (2015) Changes in standing and walking performance under dual-task conditions across the lifespan. Sports Med 45:1739-1758

11. Leone C, Feys P, Moumdjian L et al (2017) Cognitive-motor dual-task interference: a systematic review of neural correlates. Neurosci Biobehav Rev 75:348-360

12. Yogev-Seligmann G, Hausdorff JM, Giladi N (2012) Do we always prioritize balance when walking? Towards an integrated model of task prioritization. Mov Disord 27:765-770

13. Bergamin $M$, Gobbo $S$, Zanotto $T$ et al (2014) Influence of age on postural sway during different dual-task conditions. Front Aging Neurosci 6:271

14. Boisgontier MP, Beets IA, Duysens J et al (2013) Age-related differences in attentional cost associated with postural dual tasks: increased recruitment of generic cognitive resources in older adults. Neurosci Biobehav Rev 37(8):1824-1837

15. Shumway-Cook A, Woollacott M, Kerns KA et al (1997) The effects of two types of cognitive tasks on postural stability in older adults with and without a history of falls. J Gerontol A Biol Sci Med Sci 52(4):M232-M240

16. Smolders C, Doumas M, Krampe RT (2010) Posture and cognition interfere in later adulthood even without concurrent response production. Hum Mov Sci 29(5):809-819

17. Pashler H (1994) Dual-task interference in simple tasks: data and theory. Psychol Bull 116:220-244. https://doi.org/10.1037/ 0033-2909.116.2.220

18. de Dieuleveult AL, Siemonsma PC, van Erp JB et al (2017) Effects of aging in multisensory integration: a systematic review. Front Aging Neurosci 9:80

19. Stins JF, Roerdink M, Beek PJ (2011) To freeze or not to freeze? Affective and cognitive perturbations have markedly different effects on postural control. Hum Mov Sci 30:190-202

20. McNevin NH, Wulf G (2002) Attentional focus on supra-postural tasks affects postural control. Hum Mov Sci 21:187-202

21. Vuillerme N, Vincent H (2006) How performing a mental arithmetic task modify the regulation of centre of foot pressure displacements during bipedal quiet standing. Exp Brain Res 169:130-134

22. Brown LA, Polych MA, Doan JB (2006) The effect of anxiety on the regulation of upright standing among younger and older adults. Gait Posture 24:397-405

23. Morasso PG, Sanguineti V (2002) Ankle muscle stiffness alone cannot stabilize balance during quiet standing. J Neurophysiol 88:2157-2162

24. Hill CM, DeBusk H, Simpson JD et al (2019) The interaction of cognitive interference, standing surface, and fatigue on lower extremity muscle activity. Saf Health Work 10:321-326

25. Rankin JK, Woollacott MH, Shumway-Cook A, Brown LA (2000) Cognitive influence on postural stability: a neuromuscular analysis in young and older adults. J Gerontol A Biol Sci Med Sci 55:M112-M119

26. Tsuchida W, Nakagawa K, Kawahara $Y$ et al (2013) Influence of dual-task performance on muscle and brain activity. Int $\mathrm{J}$ Rehabil Res 36:127-133
27. Wuehr M, Brandt T, Schniepp R (2017) Distracting attention in phobic postural vertigo normalizes leg muscle activity and balance. Neurology 88:284-288

28. Folstein MF, Folstein SE, McHugh PR (1975) "Mini-mental state": a practical method for grading the cognitive state of patients for the clinician. J Psychiatr Res 12:189-198

29. Swan L, Otani H, Loubert PV et al (2004) Improving balance by performing a secondary cognitive task. Br J Psychol 95:31-40

30. Zok M, Mazza C, Cappozzo A (2008) Should the instructions issued to the subject in traditional static posturography be standardised? Med Eng Phys 30:913-916. https://doi.org/10.1016/j. medengphy.2007.12.002

31. Sawacha Z, Carraro E, Contessa P et al (2013) Relationship between clinical and instrumental balance assessments in chronic post-stroke hemiparesis subjects. J Neuroeng Rehabil 10:95

32. Yardley L, Gardner M, Leadbetter A et al (1999) Effect of articulatory and mental tasks on postural control. NeuroReport 10:215-219

33. Blanc Y, Dimanico U (2010) Electrode Placement in Surface Electromyography (sEMG)" Minimal Crosstalk Area “(MCA). Open Rehabil J 3(1):110-126. https://doi.org/10.2174/1874943701 003010110

34. Sacco IdCN, Amadio AC (2003) Influence of the diabetic neuropathy on the behavior of electromyographic and sensorial responses in treadmill gait. Clin Biomech 18:426-434

35. Vigotsky AD, Halperin I, Lehman GJ et al (2018) Interpreting signal amplitudes in surface electromyography studies in sport and rehabilitation sciences. Front Physiol 8:985

36. Chiari L, Rocchi L, Cappello A (2002) Stabilometric parameters are affected by anthropometry and foot placement. Clin Biomech 17:666-677

37. Raymakers J, Samson M, Verhaar H (2005) The assessment of body sway and the choice of the stability parameter (s). Gait Posture 21(1):48-58

38. Schubert P, Kirchner M, Schmidtbleicher D, Haas CT (2012) About the structure of posturography: Sampling duration, parametrization, focus of attention (part I). J Biomed Sci Eng 5(9):9. https://doi.org/10.4236/jbise.2012.59063

39. Marcolin G, Panizzolo FA, Biancato E et al (2019) Moderate treadmill run worsened static but not dynamic postural stability of healthy individuals. Eur J Appl Physiol 119:841-846

40. Streiner DL, Norman GR (2011) Correction for multiple testing: is there a resolution? Chest 140:16-18. https://doi.org/10.1378/ chest.11-0523

41. Hodges P, Gurfinkel V, Brumagne S et al (2002) Coexistence of stability and mobility in postural control: evidence from postural compensation for respiration. Exp Brain Res 144:293-302

42. Kim D, Hwang J-M (2018) The center of pressure and ankle muscle co-contraction in response to anterior-posterior perturbations. PLOS ONE 13(11):e0207667. https://doi.org/10.1371/journal. pone. 0207667

43. Maurer C, Peterka R (2005) A new interpretation of spontaneous sway measures based on a simple model of human postural control. J Neurophysiol 93:189-200

44. Rocchi L, Chiari L, Cappello A (2004) Feature selection of stabilometric parameters based on principal component analysis. Med Biol Eng Comput 42:71-79. https://doi.org/10.1007/BF023 51013

45. Cluff T, Gharib T, Balasubramaniam R (2010) Attentional influences on the performance of secondary physical tasks during posture control. Exp Brain Res 203:647-658. https://doi.org/10.1007/ s00221-010-2274-7

46. Ceyte H, Lion A, Caudron S et al (2014) Does calculating impair postural stabilization allowed by visual cues? Exp Brain Res 232:2221-2228. https://doi.org/10.1007/s00221-014-3913-1 
47. Vuillerme N, Vincent H (2005) How performing a mental arithmetic task modify the regulation of centre of foot pressure displacements during bipedal quiet standing. Exp Brain Res 169:130-134

48. Palmieri RM, Ingersoll CD, Stone MB et al (2002) Center-ofpressure parameters used in the assessment of postural control. J Sport Rehabil 11:51-66

49. Prieto TE, Myklebust JB, Hoffmann RG et al (1996) Measures of postural steadiness: differences between healthy young and elderly adults. IEEE Trans Biomed Eng 43:956-966

50. Roman-Liu D (2018) Age-related changes in the range and velocity of postural sway. Arch Gerontol Geriatr 77:68-80

51. Borg F, Finell M, Hakala I et al (2007) Analyzing gastrocnemius EMG-activity and sway data from quiet and perturbed standing. J Electromyogr Kinesiol 17:622-634
52. Duarte M, Freitas SM (2010) Revision of posturography based on force plate for balance evaluation. Braz J Phys Ther 14:183-192

53. Varrecchia T, D'Anna C, Scorza A et al (2018) Muscle activity detection in pathological, weak and noisy myoelectric signals. 2018 IEEE international symposium on medical measurements and applications (MeMeA). IEEE, pp 1-5

Publisher's Note Springer Nature remains neutral with regard to jurisdictional claims in published maps and institutional affiliations. 